\title{
Risk factors during Pregnancy and Its Relation to Preterm Pre-Labour Rupture Of Membranes
}

\author{
Ahmed , A ., A ; Al-Hussain , T ., K ; Mohamed , S ., N ; El- dien , H ., A. \\ Obstetrics and Gynecology Medicine, Assiut Faculty of Medicine \\ Obstetrics and Gynecology Nursing, Assiut Faculty of Nursing.
}

\begin{abstract}
Preterm premature rupture of the membranes (pPROM) is the rupture of membranes during pregnancy before 37 weeks of gestation. This study aimed to determine the Risk factors during Pregnancy and Its Relation to Preterm Pre-Labour Rupture Of Membranes. This prospective case-control study was carried out at the labor ward of the women's Health Center in Assiut. It included 250 pregnant women with pPROM (cases) and 250 no pPROM (controls). Data were collected using structured interview and physical assessment sheets. The results revealed that more study group women reported carrying heavy objects $(\boldsymbol{p}=\mathbf{0 . 0 4})$, using daily transportation $(\boldsymbol{p}=\mathbf{0 . 0 0 6})$, and total physical activity $(\boldsymbol{p}=\mathbf{0 . 0 4})$, more history of PROM $(\boldsymbol{p}<\mathbf{0 . 0 0 1})$, complaints $(\boldsymbol{p}<\mathbf{0 . 0 0 1})$, and sexual intercourse during third trimester $(\boldsymbol{p}=\mathbf{0 . 0 3})$. It is concluded that the risk factors of pPROM include woman's job status, residence, level of activity, previous history of PROM, having complaints during pregnancy, and having sexual intercourse during the third trimester. It is recommended that the management of pPROM include the management protocol should be improved, and strictly followed, with training of health care providers in following it. Further research is proposed to investigate the effectiveness of nursing interventions aimed at reducing the risk factors of pPROM on its occurrence.
\end{abstract}

\section{Keywords: Risk Factors - Prom- Pprom}

\section{Introduction:}

Premature rupture of membranes (PROM) is defined as rupture of the amniotic sac membranes before labor onset at 37 weeks of gestation or later. It constitutes a significant problem in obstetrics. It is termed prolonged rupture of membrane if it persists for more than 24 hours to onset of labor (Jazayeri, 2008).

Premature rupture of the amniotic sac membranes enclosing the fetus is, as yet, a not fully understood process, but may related to the mechanical properties of those membranes (Wittenberg, 2011). Umbilical cord blood cytokine values are higher than maternal levels, suggesting significant fetal/placental contribution. Maternal and umbilical cord cytokine levels are not adequately predictive to be used clinically (Mercer et al, 2012). Premature rupture of membranes occurs between 5 and $15 \%$ of pregnancies, of these, $10 \%$ occurs at term and preterm 2 to $3.5 \%$ (Hernández et al., 2011). Previable or Preterm (less than 24 weeks) premature rupture of membranes (pPROM) complicates about 1 in every thousand births and is responsible for substantial perinatal mortality (Margato et al., 2011). It is the leading identifiable cause of premature birth and accounts for approximately $18 \%$ to $20 \%$ of perinatal deaths in the United States (Caughey, 2008).

Clinical factors associated with preterm PROM include low socioeconomic status, low body mass index, tobacco use, preterm labor history, urinary tract infection, vaginal bleeding at any time in pregnancy, circulage, and amniocentesis. Fetoscopy may carry a risk of Iatrogenic pPROM, although this depends on the experience of the obstetrician (Gratacós, 2012).

Rapid diagnostic tests may be used. They are based on the detection of the insulin-like growth factorbinding protein-1 (IGFBP-1) and placental $\alpha$ microglobulin-1 (PAMG-1) in cervicovaginal secretions. AmniSure(®) test (PAMG-1) can be done

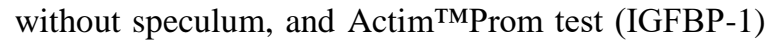
during speculum examination. Both tests have high sensitivity and specificity of about $95 \%$ (Marcellin et al., 2011).

In Egypt, maternal morbidities were strongly associated with preterm deliveries and low birth weight (LBW) even after controlling for confounders. Data indicate that Cairo and Dakahalia had the highest LBW rate reaching to $17.1 \%$ in Cairo and $14.4 \%$ in Dakahalia, Port Said and Beheira had the lowest rates (7.4 and $8.1 \%$, respectively). The overall weighted percentage of LBW was found to be $12.9 \%$. Preterm PROM is considered an obstetric problem which seems to be closely related to the occurrence of LBW, with an Odds Ratio (OR) 2.1 (Mansour et al., 2002).

Significance of the study

Preterm PROM is associated with $30-40 \%$ of preterm deliveries and is the leading identifiable cause of low birth weight. It is associated with significant risks of morbidity and mortality for both the fetus and the mother. 
Preterm delivery and LBW are considered a frequent and significant health problem in Egypt. This study was done to determine the Risk factors during Pregnancy and Its Relation to Preterm Pre-Labour Rupture Of Membranes which might be a significant risk factor for preterm delivery and LBW.

\section{Aim of the Study}

The aim of this study was to determine the Risk factors during Pregnancy and Its Relation to Preterm Pre-Labour Rupture Of Membranes.

\section{Subjects and Methods:}

\section{I.Technical Design}

The technical design includes the research design, setting of the study, subjects, and tools for data collection.

\section{Research design}

A retrospective hospital-based case-control study design was used is this study.

\section{Setting}

The study was conducted at the labor ward of the women's Health Center in Assiut. The center performs yearly approximately 6381 normal labors, and 6532 caesarean sections.

\section{Subjects}

The study subjects consisted of two groups: cases and controls, each consisting of 250 pregnant women.

- Cases group: This group included 250 pregnant women with pPROM.

- Control group: This group included 250 pregnant women with no pPROM.

Women in the two groups were similar in every respect except for pPROM. They were selected according to the following criteria:

\section{- Inclusion criteria:}

- Pregnant woman not in labor

- At 28-36 weeks of gestation

- With a singleton pregnancy.

- Exclusion criteria:

- Medical conditions necessitating elective delivery such as diabetes mellitus.

○ History of cervical insufficiency

- History of antepartum vaginal bleeding

o History of antibiotic treatment within one week

○ Polyhydramnios

- Pregnancy with major congenital anomalies.

\section{Sample size}

The sample size is estimated to detect the difference between the rate of infection in normal $\left(\mathrm{p}_{1}=40 \%\right)$ and the expected rate in the pPROM mothers $\left(\mathrm{p}_{2}=60 \%\right)$ according to Karat et al. (2006), with a $95 \%$ level of confidence $(\alpha$ error $=5 \%)$, and a study power of $80 \%$ ( $\beta$ error $=20 \%$ ). Using the equation for the difference between two proportions
(Schlesselman, 1982). Accordingly, the estimated sample size is 107 subjects per group. After adjustment for a dropout rate of $20 \%$, the sample size will be 135 women per group.

Data collection tools

Two tools were used for data collection: a structured interview questionnaire sheet, and a physical assessment sheet.

- Structured interview questionnaire sheet: It was designed by the researcher based on review of pertinent literature. It was content-validated through soliciting the opinions of experts in nursing and medical obstetrics and gynecology. The sheet included the following sections:

- Socio-demographic characteristics: such as age, education, job status, residence, income, family size and number of rooms to calculate the crowding index (number of persons per room).

- $\quad$ Risk factors and risky habits: smoking (active and passive), physical activity as carrying heavy objects, long standing, computer work, daily transportation, and sleep habits. It also included history of chronic diseases, accidents or trauma, $\mathrm{X}$-ray, amniocentesis, and sexual intercourse during the third trimester.

- Obstetric history: gravidity, parity, history of abortion, stillbirth, previous induction of labor, preterm PROM, obstructed labor, cesarean section, preterm labor, previous use of contraceptives, etc.

- $\quad$ Mode and time of last delivery.

- Details of current pregnancy: Gestational weeks, complaints, and diagnosed problems, as well as history of infections and medications during this pregnancy.

- Physical assessment sheet: This was designed by the researcher to record the physical findings during labor as well as the results of lab tests. It included data regarding:

- Vaginal examinations: number, person who did it, use of gloves and antiseptics during examination.

- Findings of examination: condition of the cervix, presenting part, condition of membranes and amniotic fluid, as well as the presence and characteristics of any vaginal discharge.

\section{Operational Design}

This design involves description of the preparatory phase, the pilot study, and then the fieldwork.

\section{Preparatory phase}

The researcher reviewed related national and international literature using textbooks, articles, and scientific journals. The tools were then prepared 
based on this literature. They were reviewed for validation by experts in obstetrics and gynecology.

\section{Pilot study}

After preparation of the tools, they were pre-tested on a sample of 50 women before the beginning of data collection to test the relevance of the questions to the aim of the work and to determine whether they are understood by the respondents or not. The researcher then did the necessary modifications in the questionnaire. The pilot study also served to determine the time needed to complete the questionnaire form. The tools were finalized based on the results of the pilot study. The pilot sample was not included in the main study sample.

\section{Fieldwork}

The field work was done during the period from $10 / 5 / 2009$ to $10 / 5 / 2010$. Upon approval of the start of the fieldwork, the researcher met with the potential women attending the center for labor. She explained the aim and procedures of the study to them, and asked for participation. Those who consented to participate in the study and were eligible according to the inclusion and exclusion criteria were interviewed using the structured questionnaire form. After completion of the interview, the researcher estimated the gestational age through the date of the Last Menstrual Period, and calculated the expected date of delivery. In case the woman does not remember the date of the Last Menstrual Period, the estimation of the gestational age was based on ultrasonography in first trimester.

Women were then asked about pPROM. Those without pPROM were included in the control group. Those with pPROM were included in the cases group. These women were asked about the time of membrane rupture, and the duration from the time of membranes rupture until the time of the interview was calculated and recorded in hours in the tool. Then, the diagnosis of pPROM was confirmed .

\section{Administrative Design}

The study protocol was approved by the ethical committee of the Faculty of Medicine at Assuit University, Women's Health Center chairman at Assiut University Hospital, and the ethical committee of the Faculty of Nursing at Assiut University. Informed oral consent was obtained before interviewing any participant, with explanation of the nature of the study to her, as well as her rights to refuse or withdraw at any time. Confidentiality was secured, and any information obtained was used only for the purpose of research.

\section{Statistical Design}

Data entry and statistical analysis were done using SPSS 16.0 statistical software package. Data were presented using descriptive statistics in the form of frequencies and percentages for qualitative variables, and means and standard deviations for quantitative variables. Quantitative continuous data were compared using the non-parametric Mann-Whitney tests since normal distribution of the data could not be assumed. Qualitative categorical variables were compared using chi-square test. Whenever the expected values in one or more of the cells in a $2 \times 2$ tables was less than 5, Fisher exact test was used instead. In larger than $2 \times 2$ cross-tables, no test could be applied whenever the expected value in $10 \%$ or more of the cells was less than 5. Statistical significance was considered at $p$-value $<0.05$. 
Table 1. Socio-demographic characteristics of pregnant women in the study and control groups

\begin{tabular}{|c|c|c|c|c|c|c|}
\hline & \multicolumn{4}{|c|}{$\overline{\text { Group }}$} & \multirow{3}{*}{$\begin{array}{c}\mathrm{X}^{2} \\
\text { Test }\end{array}$} & \multirow{3}{*}{$p$-value } \\
\hline & \multicolumn{2}{|c|}{ Study $(n=250)$} & \multicolumn{2}{|c|}{ Control $(n=250)$} & & \\
\hline & No. & $\%$ & No. & $\%$ & & \\
\hline Age (years): & & & & & & \\
\hline$<25$ & 116 & 46.4 & 110 & 44.0 & & \\
\hline $25-$ & 83 & 33.2 & 62 & 24.8 & & \\
\hline $30+$ & 51 & 20.4 & 78 & 31.2 & & \\
\hline Range & \multicolumn{2}{|c|}{$17.0-45.0$} & \multicolumn{2}{|c|}{$15.0-45.0$} & & \\
\hline Mean \pm SD & \multicolumn{2}{|c|}{$25.5 \pm 5.0$} & \multicolumn{2}{|c|}{$26.2 \pm 6.0$} & 0.92 & 0.34 \\
\hline \multicolumn{7}{|l|}{ Education: } \\
\hline Illiterate & 88 & 35.2 & 100 & 40.0 & & \\
\hline Read/write & 26 & 10.4 & 18 & 7.2 & & \\
\hline Basic & 44 & 17.6 & 46 & 18.4 & 2.86 & 0.58 \\
\hline Secondary & 76 & 30.4 & 74 & 29.6 & & \\
\hline University & 16 & 6.4 & 12 & 4.8 & & \\
\hline \multicolumn{7}{|l|}{ Job: } \\
\hline Housewife & 233 & 93.2 & 243 & 97.2 & & \\
\hline Working & 17 & 6.8 & 7 & 2.8 & 4.38 & $0.04 *$ \\
\hline \multicolumn{7}{|l|}{ Residence: } \\
\hline Urban & 63 & 25.2 & 36 & 14.4 & & \\
\hline Rural & 187 & 74.8 & 214 & 85.6 & 9.18 & $0.002 *$ \\
\hline \multicolumn{7}{|l|}{ Husband job: } \\
\hline Employee & 54 & 21.6 & 42 & 16.8 & & \\
\hline Manual worker & 196 & 78.4 & 208 & 83.2 & 1.86 & 0.17 \\
\hline \multicolumn{7}{|l|}{ Crowding index: } \\
\hline$<2$ & 127 & 50.8 & 104 & 41.6 & & \\
\hline $2+$ & 123 & 49.2 & 146 & 58.4 & 4.26 & $0.04 *$ \\
\hline
\end{tabular}

(*) Statistically significant at $p<0.05$

Table 2. Risky physical activity habits among pregnant women in the study and control groups

\begin{tabular}{|l|r|r|r|r|r|c|}
\hline \multirow{3}{*}{ Risky physical activity habits ${ }^{\circledR}$} & \multicolumn{4}{|c|}{ Group } & \multirow{2}{*}{$X^{2}$} & \multirow{2}{*}{$p$-value } \\
\cline { 2 - 6 } & \multicolumn{2}{|c|}{ Study (n=250) } & \multicolumn{2}{c|}{ Control (n=250) } & Test & \\
\cline { 2 - 6 } & No. & $\%$ & No. & $\%$ & \\
\hline Carry heavy objects & 111 & 44.4 & 88 & 35.2 & 4.42 & $0.04^{*}$ \\
\hline Long standing & 167 & 66.8 & 151 & 60.4 & 2.21 & 0.14 \\
\hline Have help at home & 126 & 50.4 & 142 & 56.8 & 2.06 & 0.15 \\
\hline Night sleep >=8 hrs & 152 & 60.8 & 153 & 61.2 & 0.01 & 0.93 \\
\hline Day sleep 1-2 hrs & 131 & 52.4 & 152 & 60.8 & 3.59 & 0.06 \\
\hline Daily computer work & 6 & 2.4 & 10 & 4.0 & 1.03 & 0.31 \\
\hline Daily transportation & 16 & 6.4 & 4 & 1.6 & 7.50 & $0.006^{*}$ \\
\hline
\end{tabular}

(@) Not mutually exclusive (*) Statistically significant at $p<0.05$ 
Table 3. Obstetric history of pregnant women in the study and control groups

\begin{tabular}{|c|c|c|c|c|c|c|}
\hline & \multicolumn{4}{|c|}{ Group } & \multirow{3}{*}{$\begin{array}{c}\mathrm{X}^{2} \\
\text { Test }\end{array}$} & \multirow{3}{*}{$p$-value } \\
\hline & \multicolumn{2}{|c|}{ Study $(n=250)$} & \multicolumn{2}{|c|}{ Control $(n=250)$} & & \\
\hline & No. & $\%$ & No. & $\%$ & & \\
\hline \multicolumn{7}{|l|}{ Gravidity: } \\
\hline 1 & 80 & 32.0 & 60 & 24.0 & & \\
\hline $2-4$ & 103 & 41.2 & 114 & 45.6 & 3.98 & 0.14 \\
\hline $5+$ & 67 & 26.8 & 76 & 30.4 & & \\
\hline \multicolumn{7}{|l|}{ Parity: } \\
\hline 0 & 93 & 37.2 & 74 & 29.6 & & \\
\hline 1 & 42 & 16.8 & 55 & 22.0 & 5.54 & 0.14 \\
\hline $2-4$ & 94 & 37.6 & 91 & 36.7 & & \\
\hline $5+$ & 21 & 8.4 & 30 & 12.0 & & \\
\hline Abortion & 72 & 28.8 & 69 & 27.6 & 0.09 & 0.77 \\
\hline Stillbirth & 13 & 5.2 & 14 & 5.6 & 0.04 & 0.84 \\
\hline \multicolumn{7}{|l|}{ No. of living children: } \\
\hline 0 & 94 & 37.6 & 81 & 32.4 & & \\
\hline 1 & 48 & 19.2 & 56 & 22.4 & 2.26 & 0.52 \\
\hline $2-4$ & 92 & 36.8 & 92 & 36.8 & & \\
\hline $5+$ & 16 & 6.4 & 21 & 8.4 & & \\
\hline \multicolumn{7}{|l|}{ Past history of:@ } \\
\hline Induction of labor & 41 & 16.4 & 38 & 15.2 & 0.14 & 0.71 \\
\hline Pre-term PROM & 17 & 6.8 & 8 & 3.2 & 3.41 & 0.06 \\
\hline Obstructed labor & 2 & 0.8 & 0 & 0.0 & Fisher & 0.50 \\
\hline PROM & 38 & 15.2 & 12 & 4.8 & 15.02 & $<0.001^{*}$ \\
\hline Cesarean & 48 & 19.2 & 57 & 22.8 & 0.98 & 0.32 \\
\hline Pre-term labor & 15 & 6.0 & 8 & 3.2 & 2.23 & 0.14 \\
\hline Miscarriage & 40 & 16.0 & 34 & 13.6 & 0.57 & 0.45 \\
\hline Elective abortion & 37 & 14.8 & 39 & 15.6 & 0.06 & 0.80 \\
\hline Circulage & 7 & 2.8 & 4 & 1.6 & 0.84 & 0.36 \\
\hline Used contraception & 102 & 40.8 & 95 & 38.0 & 0.41 & 0.52 \\
\hline \multicolumn{7}{|l|}{ Method: } \\
\hline Hormonal & 57 & 22.8 & 69 & 27.6 & 1.53 & 0.22 \\
\hline IUD & 50 & 20.0 & 49 & 19.6 & 0.01 & 0.91 \\
\hline Local & 1 & 0.4 & 0 & 0.0 & Fisher & 1.00 \\
\hline
\end{tabular}

(@) Not mutually exclusive

(*) Statistically significant at $p<0.05$

Table 4. History of exposure to infections or medications in current pregnancy among pregnant women in the study and control groups

\begin{tabular}{|l|c|c|c|c|c|l|}
\hline \multirow{2}{*}{ Current pregnancy } & \multicolumn{9}{|c|}{ Group } & \multirow{2}{*}{$\begin{array}{c}\text { X2 } \\
\text { Test }\end{array}$} & \multirow{2}{*}{$p$-value } \\
\cline { 2 - 5 } & \multicolumn{2}{|c|}{ Study $(\mathrm{n}=250)$} & \multicolumn{2}{c|}{ Control (n=250) } \\
\cline { 2 - 6 } & No. & $\%$ & No. & $\%$ & & \\
\hline Infections: & 154 & 61.6 & 167 & 66.8 & 1.47 & 0.23 \\
\hline $\begin{array}{l}\text { Types (of those with history of } \\
\text { infection): @ }\end{array}$ & & & & & & \\
\hline Vaginitis & 99 & 39.6 & 122 & 48.8 & 4.29 & $0.04^{*}$ \\
\hline Urinary tract & 22 & 8.8 & 23 & 9.2 & 0.2 & 0.88 \\
\hline Recurrent colds & 39 & 15.6 & 34 & 13.6 & 0.40 & 0.53 \\
\hline Lower respiratory & 18 & 7.2 & 16 & 6.4 & 0.13 & 0.72 \\
\hline Dermatitis & 4 & 1.6 & 0 & 0.0 & Fisher & 0.12 \\
\hline Sinusitis & 3 & 1.2 & 1 & 0.4 & Fisher & 0.62 \\
\hline
\end{tabular}




\begin{tabular}{|c|c|c|c|c|c|c|}
\hline \multirow{3}{*}{ Current pregnancy } & \multicolumn{4}{|c|}{ Group } & \multirow{3}{*}{$\begin{array}{c}\mathrm{X} 2 \\
\text { Test }\end{array}$} & \multirow{3}{*}{$p$-value } \\
\hline & \multicolumn{2}{|c|}{ Study $(n=250)$} & \multicolumn{2}{|c|}{ Control $(n=250)$} & & \\
\hline & No. & $\%$ & No. & $\%$ & & \\
\hline Otitis media & 5 & 2.0 & 5 & 2.0 & 0.00 & 1.00 \\
\hline Dental problem & 32 & 12.8 & 22 & 8.8 & 2.08 & 0.15 \\
\hline Other & 43 & 17.2 & 49 & 19.6 & 0.48 & 0.49 \\
\hline Medications: & 209 & 83.6 & 221 & 88.4 & 2.39 & 0.12 \\
\hline \multicolumn{7}{|l|}{$\begin{array}{l}\text { Types (of those on } \\
\text { medications): @ }\end{array}$} \\
\hline Cold & 17 & 6.8 & 11 & 4.4 & 1.36 & 0.24 \\
\hline Analgesics & 37 & 14.8 & 32 & 12.8 & 0.42 & 0.52 \\
\hline Antibiotics & 34 & 13.6 & 25 & 10.0 & 1.56 & 0.21 \\
\hline Antihypertensives & 10 & 4.0 & 12 & 4.8 & 0.19 & 0.66 \\
\hline Vitamins/tonics & 192 & 76.8 & 207 & 82.8 & 2.79 & 0.09 \\
\hline \multicolumn{7}{|l|}{ Others for: } \\
\hline Bleeding & 1 & 0.4 & 3 & 1.2 & & \\
\hline Vomiting & 15 & 6.0 & 29 & 11.6 & & \\
\hline Toxoplasma & 0 & 0.0 & 1 & 0.4 & -- & -- \\
\hline Diabetes & 0 & 0.0 & 1 & 0.4 & & \\
\hline Epilepsy & 1 & 0.4 & 0 & 0.0 & & \\
\hline Anticoagulant & 1 & 0.4 & 0 & 0.0 & & \\
\hline Thyroid & 1 & 0.4 & 0 & 0.0 & & \\
\hline
\end{tabular}

(@) Not mutually exclusive

(*) Statistically significant at $p<0.05$

A comparison of the socio-demographic characteristics between studied women with pPROM and control women is described in Table 1. It shows that they had similar mean age and education, with more than one-third being illiterate, $35.2 \%$ and $40.0 \%$, respectively. Although the majority of study $(93.2 \%)$ and control $(97.2 \%)$ groups were housewives, the difference was significant statistically ( $p=0.04)$. Similarly, the majority of women were from rural areas, but significantly more women in the study group were from urban areas compared to control $(p=0.002)$.

Concerning husbands' characteristics, the same table shows that they had similar distribution of jobs, with the majority being manual workers, $78.4 \%$ and $83.2 \%$, respectively. As regards socio-economic parameters, the table shows that significantly more of the study group women had crowding index less than two $(50.8 \%)$. The corresponding figure for the control group was $41.6 \%$.

Concerning physical activity, Table 2. shows only two differences of statistical significance between women in the study and control groups. These were related to carrying heavy objects $(p=0.04)$, and daily transportation $(p=0.006)$. In both differences, the activity was higher among study group women

Table 3. presents a comparison of the obstetric history between study and control groups. It shows no differences of statistical significance between the
(--) Test result not valid

two groups in their gravidity, parity, history of abortion and stillbirths, the number of living children, and the history of contraception. As regards the past history of obstetric problems, the table indicates a statistically significant difference in the history of PROM $(p<0.001)$. It is evident that more study group women had a history of such problem (15.2\%), compared to control group women $(4.8 \%)$.

Table 4. illustrates a comparison of the history of exposure to infections in current pregnancy among women in the study and control groups. It indicates no differences of statistically significance between the two groups. The only exception was the history of vaginitis, which was more frequent among women in the control group (48.8\%), compared to those in the study group $(39.6 \%), p=0.04$. As regards the history of intake of medications, the table points to no statistically significant differences.

\section{Discussion:}

The current study included 250 women with pPROM, and 250 control women without pPROM. The sociodemographic characteristics of studied women with pPROM and control women were closely similar, with a large proportion of illiterates, not working, and from rural areas. The control group had slightly but significantly more women who were housewives, and 
from rural areas. The women's husbands had also similar characteristics.

The lack of difference in the age of the present study women in the study and control group indicates that age is not a possible risk factor for pPROM. In agreement with this finding, Clearly-Goldman et al. (2005) in a large prospective multi-center study of the impact of maternal age on obstetric outcome found no significant difference in the incidence of pPROM based on maternal age after adjusting for a number of confounders. On the same line, Goldenberg et al. (2008) in a systematic review of a number of well designed predominantly retrospective cohort studies concluded that maternal age was not a significant predictor of pPROM.

However, in contradiction with this current study finding regarding women's age, Berkowitz et al. (2008) in their study about the risk factors for preterm birth found that mothers 30 years age or older had a significantly increase risk for pPROM. On the same line, a large population-based retrospective cohort study of the risks of maternal morbidity and adverse outcomes showed that increasing maternal age was associated with a significantly higher risk of pPROM after adjusting for maternal race, parity, diabetes, chronic hypertension and smoking status (Lucke and Brown, 2007). Similarly Ziadeh (2002) in a large study conducted in Jordan found that women delivering their first child at age 35 years or older were at increased risk of pPROM compared with women aged 20-29 years of age. Ferguson et al (2008) also had a similar finding regarding maternal age.

The present study could not identify any differences of statistical significance in the level of education of women in the study and control group. Therefore, education cannot be considered as a potential risk factor for pPROM. On the other hand, the study findings revealed some difference in the socioeconomic parameters of women in the study and control groups. This was evident regarding better income and lower crowding index among women in the study group. These findings are against what is expected, and is in disagreement with previous studies. Thus, Omar et al. (2005), in a prospective study carried out in Jordan, demonstrated that most women with pPROM were uneducated and belonged to lower or middle class.

Also in disagreement with the foregoing present study findings related to socio-economic level, Noor et al. (2006) in an observational study found that pPROM was more frequent among women belonging to low socioeconomic class, and those with no or low education. Similar findings were also reported by Polzin and Brady (2006) who asserted that the rates of pPROM negatively correlated with the socioeconomic level. Meanwhile, Ortiz et al. (2008) could not find any relation of statistical significance between women's socio-economic variables and the risk of having PROM.

The disagreement between the present study findings concerning the relation between the socio-economic level, including education and income might be related to that the majority of the study sample are residing in rural areas where the education and income of people have little variation, compared to urban areas. Also, the crowding index might be a less sensitive indicator of the socio-economic level in rural communities.

According to the present study, a small percentage of women in both study and control groups was working. However, this percentage was significantly higher among study group women. The finding is in congruence with Karat et al.(2006) in South India who found that the majority of women in the study and control group were housewives, but with no difference between the study and control group.

The observed differences in the present study could be related to the risk of daily transportation among working women, which may expose them to the risks of physical strains. This is confirmed by the close percentages of women who work and those exposed to daily transportation in our study. Moreover, a statistically significant difference was revealed between the study and control groups regarding exposure to daily transportation, with a higher percentage among women in the study group.

Concerning the obstetric history, it was quite similar among women in the study and control groups regarding gravidity, parity, history of abortion and stillbirths, the number of living children, and the history of contraception. These findings are in agreement with the results of the study carried out by Ekwo et al. (2008) in Chicago, which showed no significant relationship between parity and either term or preterm PROM.

Meanwhile, the current study results showed that a significantly higher percentage of women in the study group reported a history of PROM. This implies a high risk of recurrence of pPROM. The finding is in line with Lee et al. (2003) who reported a significant increase in the rates of recurrent pPROM among women in their study group. The rates of recurrence of pPROM ranged between $14.3 \%$ (Pasquier et al., 2005) and $21 \%$ (Naeye, 2008). The cause may be cervical incompetence or untreated cervico-vaginal infection by bacterial vaginosis or Chlamydia (Asrat et al., 2009).

Other possible risks during current pregnancy were investigated in the present study. The findings revealed few exposures to accidents, trauma, radiation, and amniocentesis, with no statistically 
significant difference between women in the two groups. Although these risk factors increase the risk of pPROM (Nelson et al., 2009), the lack of significant differences might be attributed to the very small numbers of women in both groups who gave a history of such exposures during their pregnancy. Meanwhile, Evaldison et al. (2001) suggested that smoking leads to changes in blood levels of micronutrients such as ascorbic acid, vitamin B12 and zinc, which may increase the risk for pPROM. Moreover, Shubert et al. (2001) clarified that nicotine causes arteriolar constriction, leading to uterine decidual ischemia and affection of the integrity of the membranes. The lack of significant association in the present study might be explained by the finding that exposure to passive smoking is highly prevalent among women in both the study and control groups, which would make it difficult to detect a statistically significant The history of last delivery among women in the study and control groups did not show any differences of statistical significance. About one-third of the women in the two groups had a history of cesarean section. The findings are consistent with a number of previous studies, which demonstrated no statistically significant associations between the mode of previous delivery and the risk of pPROM (Al-Qa' $Q a^{\prime}$ and $\boldsymbol{A l}$ Awaysheh, 2005; Noor et al., 2006; AngkharnTriniti et al., 2008).

Concerning the obstetric history, it was quite similar among women in the study and control groups regarding gravidity, parity, history of abortion and stillbirths, the number of living children, and the history of contraception. These findings are in agreement with the results of the study carried out by Ekwo et al. (2008) in Chicago, which showed no significant relationship between parity and either term or preterm PROM. On the same line, Omar et al. (2005) in their study in Jordan could not reveal any statistically significant associations between pPROM and parity. However, Newman et al. (2001) reported that the prevalence of pPROM among multiparous women was substantially higher than among nulliparous women. In the present study, the percent of nulliparous women was indeed higher in the study group than in the control group, but the difference could not reach statistical significance.

\section{Conclusion:}

The study concludes that the pre-term premature rupture of membranes (pPROM) among pregnant women may have some associated risk factors. It may be related to some socio-demographic characteristics as woman's job status, and residence. It is also influenced by the level of activity of the woman. The obstetric risk factors include a previous history of PROM, having complaints during pregnancy, and having sexual intercourse during the third trimester.

\section{Recommendations:}

In view of the study findings, the following recommendations are proposed.

- The management of pPROM must include a cervical swab with culture sensitivity, and proper antibiotics should be used;

- The management protocol should be improved accordingly, and strictly followed in order to improve neonatal outcomes; this should be under close supervision according to the protocol;

- There is a need to train health care providers, including doctors and nurses in maternal and childcare services, on following this protocol, and to provide them with sound and reliable information on management of pPROM.

- Special care should be given to women with a history of PROM and those having complaints during their current pregnancy;

- Health education programs about the risk factors for pPROM during pregnancy should be incorporated in antenatal care services, with emphasis on the risk of sexual intercourse without proper hygiene during the third trimester;

- Policy-makers should be aware of the importance and seriousness of the problem of pPROM and its cost to the healthcare system budget;

- Further research is proposed to investigate the effectiveness of nursing interventions aimed at reducing the risk factors of $\mathrm{pPROM}$ on its occurrence.

\section{References :}

1. Artal R., Burgeson R., Fernandes F.J. and Hobel C.J. (2009): Fetal and maternal copper levels in patients at term with and without premature rupture of membrane. Obstetric Gynecology; 53:608-610.

2. Asrat T., Lewis D.F., and Garite T.J. (2009): Rate of recurrence of preterm premature rupture of membrane in consecutive pregnancies. American Journal Obstetric and Gynecology; 165: 1111-1115.

3. Berkowitz G., Blackmore-prince C., Lapinski R., and Savitz D. (2008): Risk factors for preterm birth. Epidemiology; 9:279-285.

4. Caughey A (2008): Contemporary Diagnosis and Management of Preterm Premature Rupture of Membranes. Obstetric Gynecology; 1(1): 1122. 
5. Cle Hernández y Ballinas A, López Farán JA, Gámez Guevara C. (2011): [Comparison of maternal and perinatal outcomes in the conservative treatment preterm premature membrane rupture between the use of erythromycin and clindamycin]. [Article in Spanish]. Ginecol Obstet Mex.;79(7):403-10

6. Arly-Goldman J., Malone F., Vidaver J., Hall R., Nyberg D.,Cormstock C.(2005): Impact of maternal age on obstetric outcome. Obstetric Gynecology; 105:983-990.

7. Ekwo E., Gosselink C.A., Woolson R.F., and Atef Moawa D.A. (2008): Risks for premature rupture of amniotic membranes. International Journal of Epidemiology; 22(3):495-503.

8. Ferguson S., Salenieks M., Windrim R., and Walker M. (2008): pPROM. Nutrition and socioeconomic factors. Obstetric Gynecology; 100(6): 1250-1256.

9. Goldenberg R.L., Hauth J.C., and Andrews W.W. (2008): pPROM and Intrauterine infection. N Engl J Med.; 342:1500-1557.

10. Gratacós E. (2012): Fetoscopy and Risk of Iatrogenic Preterm Premature Rupture of Membranes: Not as High as It May Seem (in Experienced Hands). Fetal Diagn Ther.; 31(1):10-11.

11. Hernández y Ballinas A, López Farán JA, Gámez Guevara C. (2011): [Comparison of maternal and perinatal outcomes in the conservative treatment preterm premature membrane rupture between the use of erythromycin and clindamycin]. [Article in Spanish]. Ginecol Obstet Mex.;79(7):403-10

12. Jazayeri A (2008): Premature rupture of membrane. Medicine Clinical Reference; 1-11.

13. Lee T., Carpenter M., Heber W., and Silver H. (2003): Preterm premature rupture of membranes: risks of recurrent complications in the next pregnancy among a populationbased sample of gravid women. Am J Obstet Gynecol; 188(1): 209-13.

14. Lucke B., and Brown M. (2007): Risks of maternal morbidity and adverse outcomes with increase maternal age and plurality. Fertil. Steril.; 88(2):283-293.

15. Karat C., Madhivanan P.,Krupp K.., Poornima S.,Jayanthi N.V., Suguna J.S., and Mathai E. (2006)) Mansour E (2002)

16. The clinical and microbiological correlates of premature rupture of membrane. Indian Journal of Medical Microbiology; 24(4):283-285.

17. Incidence and factors leading to low birth weight in Egypt. International Pediatrics; 17:4

18. Marcellin L, Anselem O, Guibourdenche J, De la Calle A, Deput-Rampon C, Cabrol D,
Tsatsaris V. (2011): [Comparison of two bedside tests performed on cervicovaginal fluid to diagnose premature rupture of membranes]. [Article in French]. J Gynecol Obstet Biol Reprod (Paris).; 40(7):651-6.

19. Margato MF, Martins GL, Passini Júnior R, Nomura ML.(2011): Previable preterm rupture of membranes: gestational and neonatal outcomes. Arch Gynecol Obstet. 2011 Dec 28. [Epub ahead of print]

20. McDonald H.M., O'Loughlin J.A., Jolley P.T., Vigneswaran R., and McDonald P.J. (2010): Changes in vaginal flora during pregnancy and association with preterm birth. J Infect Disease; 170:724-728.

21. McGregor J.A., French J.I., Lawellin D., Franco-Buff A., Smith C., and Todd J.K. (2001): Bacterial protease-induced reduction of chorioamniotic membrane strength and elasticity. Obstetric Gynecology; 69:167-174.

22. Mercer BM, Crouse DT, Goldenberg RL, Miodovnik M, Mapp DC, Meis PJ, Dombrowski MP (2012): The antibiotic treatment of pPROM study: systemic maternal and fetal markers and perinatal outcomes. Am J Obstet Gynecol.; 206

23. Mercer BM, Crouse DT, Goldenberg RL, Miodovnik M, Mapp DC, Meis PJ, Dombrowski MP (2012): The antibiotic treatment of pPROM study: systemic maternal and fetal markers and perinatal outcomes. Am J Obstet Gynecol.; 206(2):145.e1-9

24. Naeye R. (2008): Factors that predispose to premature rupture of the fetal membrane. Obstetric Gynecology; 60(1):93-98.

25. Omar M.D., Ebrahim Ayyad M.D., Hussam Nababteh M.D. (2005): Preterm Premature Rupture of Membranes (pPROM): Incidence and Outcome. Journal of the Bahrain Medical Society; 17(1):20-25.

26. Pasquier J., Rabilloud M., Picaud J., Ecochard R., Claris O., and Gaucherand P. (2005): Aprospective population based study of 598 cases of pPROM between 24 and 34 weeks gestation: description, management and mortality.

27. Schlesselman J. (1982): Case control studies: design, conduct, analysis. Oxford Uni. Press, New York, pp 145-146.

28. Simhan H.N., and Canavan T.P. (2008): Preterm premature rupture of membranes: diagnosis, evaluation and management strategies. BJOG; 112: 1-32

29. Simhan HN, Canavan TP (2005): Preterm premature rupture of membranes: diagnosis, 
evaluation and management strategies. BJOG; 112 Suppl 1:32-7

30. Wittenberg GF. (2011): Elastic properties and yield stress of fetal membranes. Conf Proc IEEE Eng Med Biol Soc.; 2123-6

31. Ziadeh S. (2002): Maternal and prenatal outcome in nulliparous women aged 35 and older. Gynecology Obstetric Invest.; 54(1):6-10. 